Steven Fox, MD

Critical Care Medicine,

Respiratory Institute, Cleveland Clinic
Rishik Vashisht, MD

Critical Care Medicine,

Respiratory Institute, Cleveland Clinic
Matthew Siuba, DO

Critical Care Medicine,

Respiratory Institute, Cleveland Clinic
Siddharth Dugar, MD

Pulmonary Medicine,

Respiratory Institute, Cleveland Clinic, Assistant Professor of Medicine, Cleveland

Clinic Lerner College of Medicine of Case

Western Reserve University

\title{
Evaluation and management of shock in patients with COVID-19
}

\section{Posted July 2, 2020}

\section{ABSTRACT}

Shock is common in critically ill patients with COVID-19, developing in up to $67 \%$ of patients in intensive care ( $5 \%$ to $10 \%$ overall) and is associated with high mortality. Optimal management requires prompt recognition with precise evaluation and differentiation. Correcting hypoperfusion and treating the underlying process are fundamental aspects of treatment. Undifferentiated shock may be treated initially with norepinephrine to optimize perfusion while additional evaluation is performed to categorize the shock pathophysiology. Physical examination, bedside echocardiography, hemodynamic monitoring, lactate and venous oxygen saturation are important components of the patient evaluation.

\section{INTRODUCTION}

Shock is a clinical state of circulatory failure characterized by impaired oxygen delivery or utilization at the cellular level. ${ }^{1}$ The basic characteristics of shock usually consist of systemic hypotension (systolic blood pressure $<90 \mathrm{mmHg}$ or mean arterial pressure $<65 \mathrm{mmHg}$ ), organ hypoperfusion, and abnormal cellular oxygen metabolism. Between $5 \%$ and $10 \%$ of the patients infected with severe acute respiratory syndrome coronavirus 2 (SARS-CoV-2) require intensive care unit (ICU) admission with up to $67 \%$ developing shock. ${ }^{2}$ Shock has been implicated as the primary cause of death in $7 \%$ of coronavirus disease 2019 (COVID-19) cases and as a contributing factor in an additional 33\%. All 4 types of shockdistributive, cardiogenic, obstructive, and hypovolemic shock-have been observed in patients with COVID-19. ${ }^{4}$

The statements and opinions expressed in COVID-19 Curbside Consults are based on experience and the available literature as of the date posted. While we try to regularly update this content, any offered recommendations cannot be substituted for the clinical judgment of clinicians caring for individual patients.

doi:10.3949/ccjm.87a.ccc052
This review discusses the classification and management of shock in patients with COVID-19. The most common aspects of evaluation are detailed in Table 1. The common causes and characteristics of shock are listed in Table 2. Advanced and invasive hemodynamic monitoring techniques may be needed if conventional techniques fail to diagnose the cause of shock or for hemodynamic monitoring. The increased risk of thromboembolic events in this group has to be calculated when considering advance invasive monitoring devices.

\section{DISTRIBUTIVE SHOCK}

Septic shock appears to be the predominant cause of distributive shock in patients with COVID-19, secondary to the virus itself or from bacterial coinfections. COVID-19 has also been associated with a hyper-inflammatory immune response with elevated cytokine levels termed cytokine storm or cytokine release syndrome. This syndrome leads to loss of vasomotor tone and higher rates of mortality. The occasional greater need for sedation in severe cases of COVID-19-associated pneumonia may cause hypotension from direct medication effect or blunted sympathetic drive.

There is limited information on the presence of bacterial coinfection with COVID-19-associated pneumonia. Extrapolating data from Middle East respiratory syndrome (MERS) and influenza showing that $18 \%$ and $11 \%$ of patients developed bacterial co-infection, respectively, ${ }^{2,5}$ may provide support for initiating empiric antibiotics in critically ill COVID19 patients. It is also prudent to practice antibiotic stewardship by rapidly discontinuing antibiotics if no evidence of bacterial infection is found.

The choice of an empiric regimen should be based on the patient's clinical history and local microbiological characteristics. Among hospitalized patients with COVID-19 who require supplemental oxygen, current IDSA guidelines recommend a 5-day course of remdesivir. ${ }^{6}$ The use of other COVID-19-specific 
TABLE 1

\section{Key parameters in the evaluation of shock}

Physical examination

Capillary refill

Mental status

Extremity temperature

Urine output

Focused echocardiography

LV systolic function

RV size and function

LVOT VTI

Pericardial effusion

Mitral, aortic, and tricuspid valve assessment

Regional wall motion abnormality

\section{Laboratory studies}

Mixed venous oxygen saturation

Central venous oxygen saturation

Lactate

$\mathrm{LV}=$ left ventricle; LVOT VTI = left ventricular outflow tract velocity time integral; $\mathrm{RV}=$ right ventricle

antimicrobial therapy remains an area of active clinical research and their use in routine clinical practice cannot be recommended at this time point.

The evidence on sepsis and acute respiratory distress syndrome (ARDS) suggests using conservative fluid management over a liberal management strategy. ${ }^{7,8}$ In patients with ARDS, a conservative strategy resulted in increased ventilator-free and ICU-free days with reduced need for renal replacement therapy (RRT) versus a liberal strategy. ${ }^{8}$ If needed, balanced crystalloids are the preferred resuscitative fluid owing to its potential reno-protective effect, which reduces the need for RRT as well as providing a possible mortality benefit. ${ }^{9}$ Other evidence suggests use of dynamic indices for fluid responsiveness (eg, pulse pressure variation, systolic pressure variation, passive leg raise, and end-expiratory occlusion tests) over static measures (eg, central venous pressure). ${ }^{10-12}$ A strategy of fluid resuscitation based on preload responsiveness has shown improved outcomes with reduced need for RRT and mechanical ventilation. ${ }^{13}$

Norepinephrine remains the preferred vasopressor agent in septic shock. Vasopressin or epinephrine may be added as a second agent if distributive shock remains the predominant component. ${ }^{2}$ There is an increasing body of evidence suggesting improved outcomes with early administration of vasopressors. ${ }^{14,15}$
If there is concern for inadequate cardiac output, an inotropic agent may be added. Stress-dosed steroids are routinely used in septic shock if perfusion remains impaired despite the above resuscitation strategies and may have a role in patients with acute respiratory failure requiring invasive mechanical ventilation. ${ }^{16}$

The current recommendation is to target mean arterial pressure (MAP) goals of 60 to $65 \mathrm{mmHg} .{ }^{2} \mathrm{An}$ initial goal of MAP above $65 \mathrm{mmHg}$, which is later individualized based on a dynamic assessment of perfusion using capillary refill time, mentation and urine output, is a reasonable approach. ${ }^{17,18}$

\section{CARDIOGENIC SHOCK}

The incidence of acute cardiac injury (defined by an elevated troponin level) in patients with COVID19 ranges from $20 \%$ to $30 \%$ and is associated with increased mortality. ${ }^{19,20}$ Causes of acute cardiac injury include demand ischemia, myocarditis, stress-induced cardiomyopathy, or less commonly, acute plaque rupture that may present as single or biventricular failure. ${ }^{19,21}$ Severe left ventricular systolic failure has been reported in COVID-19 from myocarditis, ${ }^{22}$ ST-elevation myocardial infarction (STEMI) with systolic heart failure and shock, ${ }^{23}$ or from worsening of underlying cardiovascular disease. ${ }^{21}$ Right ventricle failure may develop from pulmonary embolism or from pulmonary hypertension (ie, acute cor pulmonale) due to hypoxia, hypercapnia, and/or high mean airway pressures.

These patients should be carefully monitored for significant cardiac injury and development or worsening of shock. At this point, repeat echocardiograms, NT-proBNP levels, troponin levels, and focused cardiac ultrasound should be pursued. The clinical and echocardiographic characteristics of cardiogenic shock are presented in Table 2 .

Norepinephrine is first-line vasopressor in patients with cardiogenic shock who are hypotensive, albeit with limited data. ${ }^{24}$ If the patient continues to have severely reduced cardiac output with signs of organ hypoperfusion, as evident by clinical, laboratory, and echocardiographic examination (cold extremities, low central venous oxygen saturation, left or right ventricle systolic dysfunction on echocardiography), inotropic agents such as dobutamine or epinephrine may be considered. ${ }^{24}$

Right ventricle failure in ARDS is common and under-recognized, with reported incidence rates ranging from $25 \%$ to $50 \% .{ }^{25,26}$ Development of right ventricle failure from ARDS or injurious ventilator 
TABLE 2

Common shock etiologies and differentiation strategies

\begin{tabular}{|c|c|c|c|c|c|c|}
\hline $\begin{array}{l}\text { Type of } \\
\text { shock }\end{array}$ & Etiologies & Extremities & $\begin{array}{l}\text { Cardiac } \\
\text { output }\end{array}$ & $\begin{array}{l}\text { Mixed } \\
\text { venous } \mathrm{O}_{2}\end{array}$ & $\begin{array}{l}\text { LV systolic } \\
\text { function }\end{array}$ & $\begin{array}{l}\text { RV size/ } \\
\text { function }\end{array}$ \\
\hline Distributive & $\begin{array}{l}\text { Sepsis }^{\mathrm{a}} \\
\text { Cytokine storm }^{\mathrm{a}} \\
\text { Medication-related vasoplegia } \\
\text { Anaphylaxis } \\
\text { Neurogenic }\end{array}$ & $\begin{array}{l}\text { Warm } \\
\text { (sometimes } \\
\text { cold) }\end{array}$ & $\begin{array}{l}\text { Normal } \\
\text { or high }\end{array}$ & $\begin{array}{l}\text { Normal } \\
\text { or high }\end{array}$ & $\begin{array}{l}\text { Normal } \\
\text { or high }\end{array}$ & Normal \\
\hline \multirow[t]{3}{*}{ Cardiogenic } & $\begin{array}{l}\text { Pre-existing heart disease } \\
\text { Acute myocardial ischemia } \\
\text { Cardiomyopathy } \\
\text { Acute myocarditis }\end{array}$ & Cold & Low & Low & Low & $\begin{array}{l}\text { Normal or dilated/ } \\
\text { reduced function }\end{array}$ \\
\hline & Acute valvular disease & Cold & Low & Low & $\begin{array}{l}\text { Normal or } \\
\text { hyperdynamic }\end{array}$ & Variable \\
\hline & Right ventricle failure $^{a}$ & Cold & Low & Low & $\begin{array}{l}\text { Normal or } \\
\text { hyperdynamic }\end{array}$ & $\begin{array}{l}\text { Dilated/ } \\
\text { reduced function }\end{array}$ \\
\hline \multirow[t]{2}{*}{ Obstructive } & Pulmonary embolisma & Cold & Low & Low & $\begin{array}{l}\text { Normal or } \\
\text { hyperdynamic }\end{array}$ & $\begin{array}{l}\text { Dilated/ } \\
\text { reduced function }\end{array}$ \\
\hline & $\begin{array}{l}\text { Dynamic hyperinflation (auto-PEEP) } \\
\text { Pericardial tamponade } \\
\text { Abdominal compartment syndrome } \\
\text { Pneumothorax }\end{array}$ & Cold & Low & Low & $\begin{array}{l}\text { Normal or } \\
\text { hyperdynamic }\end{array}$ & Normal \\
\hline Hypovolemic & $\begin{array}{l}\text { Volume depletion } \\
\text { Hemorrhage }\end{array}$ & Cold & Low & Low & $\begin{array}{l}\text { Normal or } \\
\text { hyperdynamic }\end{array}$ & Normal \\
\hline
\end{tabular}

$\mathrm{LV}=$ left ventricle; RV = right ventricle

${ }^{a}$ Common in COVID-19.

settings should be managed with "right ventricle protective ventilation," which primarily involves minimizing airway pressures (optimizing positive endexpiratory pressure [PEEP], driving pressure, plateau pressure) and prone position ventilation. ${ }^{27,28}$ There are physiologic rationales for using inhaled pulmonary vasodilators (eg, epoprostenol or nitric oxide) to reduce pulmonary vascular resistance, augment right ventricle performance, and improve pulmonary ventilation and perfusion (V/Q) matching..$^{29}$

Refractory cardiogenic shock may prompt consideration of mechanical circulatory support, including veno-arterial extra corporeal life support. A guidance document on use of extracorporeal membrane oxygenation in COVID-19 patients has been published..$^{30}$ This should be discussed by an experienced, multidisciplinary team at a qualified ECMO center.

\section{OBSTRUCTIVE SHOCK}

COVID-19 has been linked to coagulopathy and increased thrombotic risk. The incidence varies from
$25 \%$ to $50 \%$, with some thrombotic events occurring despite adequate prophylactic or therapeutic anticoagulation. ${ }^{31,32}$ In one study, acute pulmonary embolism (PE) accounted for $81 \%$ of all acute thrombotic complications in patients with COVID-19.33 In autopsies performed on 12 patients, acute PE was found to be the direct cause of death in 4 patients. ${ }^{34}$

Acute PE should be suspected in patients with sudden hemodynamic or respiratory deterioration. The presence of acute PE as the cause of shock is an indication for systemic thrombolysis. ${ }^{35}$ Full therapeutic anticoagulation is indicated in the presence of any acute venous thromboembolism.

During the COVID-19 pandemic, many centers have adopted protocols for "enhanced prophylaxis" using higher than normal doses of thromboprophylaxis in patients with elevated D-dimer levels. ${ }^{36,37}$ Based on expert opinion, the Anticoagulation Forum (a North American organization of anticoagulation providers) recommended using high-intensity prophylaxis (eg, enoxaparin $40 \mathrm{mg}$ subcutaneous twice 
daily, enoxaparin $0.5 \mathrm{mg} / \mathrm{kg}$ subcutaneous twice daily, heparin 7500 units subcutaneous three times daily, or low-intensity heparin infusion). ${ }^{38}$ However, recently published Chest guidelines recommend standarddose thromboprophylaxis in critically ill COVID19 patients due to lack of evidence on the bleeding risks. ${ }^{39}$

Dynamic hyperinflation can be commonly seen in patients with ARDS who are ventilated with high respiratory rates due to inadequate expiratory time. In the setting of high airway resistance, this may lead to dynamic hyperinflation with elevated intrinsic PEEP (or auto PEEP). High airway resistance may occur due to mucous plugging in the airways, including the endotracheal tube. Routine ventilator checks should include assessment of intrinsic PEEP and airway resistance. Other management strategies might include bronchodilators, hypertonic saline, neuromuscular blockade, airway clearance, use of a large $(\mathrm{eg}, 8.0 \mathrm{~mm})$ endotracheal tube when able, and heated humidity for ventilator circuits. None of these techniques have been prospectively evaluated for efficacy.

Pneumothorax may occur due to injurious airway pressures and is an important diagnosis to consider during acute decompensation. Needle decompression may be considered in acute hemodynamic decompensation setting due to pneumothorax Management includes tube thoracostomy, typically with a medium bore pigtail catheter.

Finally, although rare, cardiac tamponade has been reported in at least 1 COVID-19 case as a result of a hemorrhagic etiology. ${ }^{40}$ As part of a focused cardiac ultrasound, pericardial effusions should be evaluated for hemodynamic significance, bearing in mind that tamponade features on echocardiography need to be correlated with a clinical examination.

\section{HYPOVOLEMIC SHOCK}

Hypovolemia may be present in earlier stages of hospitalization due to poor oral intake and high-grade fever causing insensible losses, possibly along with associated diarrhea. The benefit of intravenous fluids administration for dehydration should be weighed against concern for worsening pulmonary edema and hypoxemia, further supporting the use of preload responsiveness assessment to guide fluid administration. Bleeding is another potential cause and may occur due to high rates of coagulopathy and associated anticoagulation use.

There are no COVID-19 specific transfusion recommendations; however, a hemoglobin goal of greater than $7.0 \mathrm{mg} / \mathrm{dL}$ is appropriate based on prior data for patients with sepsis and critical illness. ${ }^{41}$ Additional blood products (fresh frozen plasma, platelets, cryoprecipitate) can be used as appropriate in the setting of coagulopathy and need for massive transfusion.

\section{OTHER CONSIDERATIONS}

Frequent contact with the patient, though a cornerstone in attentive shock resuscitation, must be minimized in COVID-19 cases to reduce healthcare worker viral exposure and personal protective equipment use. There are several ways to reduce patient contact. Central venous pressure waveforms and pulse pressure variation can be displayed on the patient monitor as additional continuous monitoring variables. Mental status can be assessed through telecommunication. Intravenous pumps can be relocated outside the room with tubing extensions to permit bedside nurses to efficiently titrate vasoactive medications from outside the room. Obtaining central and arterial access early can reduce overall exposure of healthcare workers to the patient. Using a protocolized point-of-care ultrasound can eliminate the need for a consultative complete echocardiogram and associated human and equipment exposure. ${ }^{42}$ Nevertheless, direct contact is still required to assess capillary refill and extremity warmth, and it is ideally done when other care is being provided (ie, bundled care).

\section{SUMMARY}

Shock of a variety of etiologies is common in critically ill patients with COVID-19 and is associated with high mortality. Prompt recognition along with precise evaluation and management are keys to patient care. Correcting hypoperfusion and treatment of the underlying process are the fundamental aspects of management. Undifferentiated shock may be managed initially with norepinephrine to optimize perfusion while urgent evaluation is performed to categorize the shock pathophysiology. Physical examination, bedside echocardiography, hemodynamic monitoring, and central venous oxygen saturation are important components of evaluation. Further studies are needed to clarify whether there are any nuances to COVID-19-associated shock management, particularly with distributive shock, as compared with other shock etiologies. 


\section{REFERENCES}

1. Vincent J-L, De Backer D. Circulatory shock. N Engl J Med 2013; 369(18):1726-1734. doi:10.1056/NEJMra1208943

2. Alhazzani W, Møller MH, Arabi YM, et al. Surviving sepsis campaign: guidelines on the management of critically ill adults with coronavirus disease 2019 (COVID-19). Crit Care Med 2020; 48(6):e440-e469. doi:10.1097/CCM.0000000000004363

3. Ruan Q, Yang K, Wang W, Jiang L, Song J. Clinical predictors of mortality due to COVID-19 based on an analysis of data of 150 patients from Wuhan, China. Intensive Care Med 2020; 46(5):846-848. doi:10.1007/s00134-020-05991-x

4. Zhang C, Wu Z, Li JW, Zhao H, Wang GQ. Cytokine release syndrome in severe COVID-19: interleukin-6 receptor antagonist tocilizumab may be the key to reduce mortality. Int J Antimicrob Agents 2020; 55(5):105954. doi:10.1016/j.ijantimicag.2020.105954

5. Uyeki TM, Bernstein HH, Bradley JS, et al. Clinical practice guidelines by the Infectious Diseases Society of America: 2018 update on diagnosis, treatment, chemoprophylaxis, and institutional outbreak management of seasonal influenza. Clin Infect Dis 2019; 68(6):e1e47. doi:10.1093/cid/ciy866

6. Bhimraj A, Morgan RL, Shumaker AH, et al. Infectious Diseases Society of America Guidelines on the treatment and management of patients with COVID-19. Clin Infect Dis 2020; Apr 27 [updated June 22]. doi:10.1093/cid/ciaa478

7. Silversides JA, Major E, Ferguson AJ, et al. Conservative fluid management or deresuscitation for patients with sepsis or acute respiratory distress syndrome following the resuscitation phase of critical illness: a systematic review and meta-analysis. Intensive Care Med 2017; 43(2):155-170. doi:10.1007/s00134-016-4573-3

8. National Heart, Lung, and Blood Institute Acute Respiratory Distress Syndrome (ARDS) Clinical Trials Network, Wiedemann HP, Wheeler AP, et al. Comparison of two fluid-management strategies in acute lung injury. N Engl J Med 2006; 354(24):2564-2575. doi:10.1056/NEJMoa062200

9. Semler MW, Self WH, Wanderer JP, et al. Balanced crystalloids versus saline in critically ill adults. N Engl J Med 2018; 378(9):829-839. doi:10.1056/NEJMoa1711584

10. Bednarczyk JM, Fridfinnson JA, Kumar A, et al. Incorporating dynamic assessment of fluid responsiveness into goal-directed therapy: a systematic review and meta-analysis. Crit Care Med 2017; 45(9):1538-1545. doi:10.1097/CCM.0000000000002554

11. Monnet X, Marik $P$, Teboul J-L. Passive leg raising for predicting fluid responsiveness: a systematic review and meta-analysis. Intensive Care Med 2016; 42(12):1935-1947. doi:10.1007/s00134-015-4134-1

12. Cherpanath TG, Hirsch A, Geerts BF, et al. Predicting fluid responsiveness by passive leg raising: a systematic review and meta-analysis of 23 clinical trials. Crit Care Med 2016; 44(5):981-991. doi:10.1097/ CCM.0000000000001556

13. Douglas IS, Alapat PM, Corl KA, et al. Fluid response evaluation in sepsis hypotension and shock: a randomized clinical trial. Chest 2020; May. doi:10.1016/j.chest.2020.04.025

14. Permpikul C, Tongyoo S, Viarasilpa T, Trainarongsakul T, Chakorn T, Udompanturak S. Early use of norepinephrine in septic shock resuscitation (CENSER). a randomized trial. Am J Respir Crit Care Med 2019; 199(9):1097-1105. doi:10.1164/rccm.201806-1034OC

15. Ospina-Tascón GA, Hernandez G, Alvarez I, et al. Effects of very early start of norepinephrine in patients with septic shock: a propensity score-based analysis. Crit Care 2020; 24(1):52. doi:10.1186/ s13054-020-2756-3

16. Villar J, Ferrando C, Martínez D, et al. Dexamethasone treatment for the acute respiratory distress syndrome: a multicentre, randomised controlled trial. Lancet Respir Med 2020; 8(3):267-276. doi:10.1016/S2213-2600(19)30417-5

17. Dugar S, Choudhary C, Duggal A. Sepsis and septic shock: guideline-based management. Cleve Clin J Med 2020; 87(1):53-64. doi:10.3949/ccjm.87a.18143

18. Hernández G, Ospina-Tascón GA, Damiani LP, et al. Effect of a resuscitation strategy targeting peripheral perfusion status vs serum lactate levels on 28-day mortality among patients with septic shock: the ANDROMEDA-SHOCK randomized clinical trial. JAMA 2019; 321(7):654-664. doi:10.1001/jama.2019.0071

19. Akhmerov A, Marbán E. COVID-19 and the heart. Circ Res 2020; 126(10):1443-1455. doi:10.1161/CIRCRESAHA.120.317055

20. Shi S, Qin M, Shen B, et al. Association of cardiac injury with mortality in hospitalized patients with COVID-19 in Wuhan, China. JAMA Cardiol 2020; Mar 25. doi:10.1001/jamacardio.2020.0950

21. Madjid M, Safavi-Naeini P, Solomon SD, Vardeny O. Potential effects of coronaviruses on the cardiovascular system: a review. JAMA Cardiol 2020; Mar 27. doi:10.1001/jamacardio.2020.1286

22. Inciardi RM, Lupi L, Zaccone G, et al. Cardiac involvement in a patient with coronavirus disease 2019 (COVID-19). JAMA Cardiol 2020; Mar 27. doi:10.1001/jamacardio.2020.1096

23. Bangalore S, Sharma A, Slotwiner A, et al. ST-segment elevation in patients with Covid-19 - a case series. N Engl J Med 2020; Apr 17. doi:10.1056/NEJMc2009020

24. van Diepen S, Katz JN, Albert NM, et al. Contemporary management of cardiogenic shock: a scientific statement from the American Heart Association. Circulation 2017; 136(16):e232-e268. doi:10.1161/CIR.0000000000000525

25. Vieillard-Baron $\mathbf{A}$. Is right ventricular function the one that matters in ARDS patients? Definitely yes. Intensive Care Med 2009; 35(1):4-6. doi:10.1007/s00134-008-1308-0

26. Mekontso Dessap A, Boissier F, Charron C, et al. Acute cor pulmonale during protective ventilation for acute respiratory distress syndrome: prevalence, predictors, and clinical impact. Intensive Care Med 2016; 42(5):862-870. doi:10.1007/s00134-015-4141-2

27. Vieillard-Baron A, Charron C, Caille V, Belliard G, Page B, Jardin F. Prone positioning unloads the right ventricle in severe ARDS. Chest 2007; 132(5):1440-1446. doi:10.1378/chest.07-1013

28. Zochios V, Parhar K, Vieillard-Baron A. Protecting the right ventricle in ARDS: the role of prone ventilation. J Cardiothorac Vasc Anesth 2018; 32(5):2248-2251. doi:10.1053/j.jvca.2018.01.007

29. Searcy RJ, Morales JR, Ferreira JA, Johnson DW. The role of inhaled prostacyclin in treating acute respiratory distress syndrome. Ther Adv Respir Dis 2015; 9(6):302-312. doi:10.1177/1753465815599345

30. Bartlett RH, Ogino MT, Brodie D, et al. Initial ELSO guidance document: ECMO for COVID-19 patients with severe cardiopulmonary failure. ASAIO J 2020; 66(5):472-474.

31. Llitjos JF, Leclerc M, Chochois C, et al. High incidence of venous thromboembolic events in anticoagulated severe COVID-19 patients. J Thromb Haemost 2020; Apr 22. doi:10.1111/jth. 14869

32. Helms J, Tacquard C, Severac F, et al. High risk of thrombosis in patients with severe SARS-CoV-2 infection: a multicenter prospective cohort study. Intensive Care Med 2020; 46(6):1089-1098. doi:10.1007/s00134-020-06062-x

33. Klok FA, Kruip MJHA, van der Meer NJM, et al. Incidence of thrombotic complications in critically ill ICU patients with COVID-19. Thromb Res 2020; 191:145-147. doi:10.1016/j.thromres.2020.04.013

34. Wichmann D, Sperhake J-P, Lütgehetmann M, et al. Autopsy findings and venous thromboembolism in patients with COVID-19. Ann Intern Med 2020; May. doi:10.7326/M20-2003

35. Chatterjee S, Chakraborty A, Weinberg I, et al. Thrombolysis for pulmonary embolism and risk of all-cause mortality, major bleeding, and intracranial hemorrhage: a meta-analysis. JAMA 2014; 311(23):2414-2421. doi:10.1001/jama.2014.5990

36. Mucha SR, Dugar S, McCrae K, et al. Coagulopathy in COVID-19. Cleve Clin J Med 2020; Jun 10. doi:10.3949/ccjm.87a.ccc024

37. Paranjpe I, Fuster V, Lala A, et al. Association of treatment dose anticoagulation with in-hospital survival among hospitalized patients with COVID-19. J Am Coll Cardiol 2020; May 5. doi:10.1016/j. jacc.2020.05.001

38. Barnes GD, Burnett A, Allen A, et al. Thromboembolism and anticoagulant therapy during the COVID-19 pandemic: interim clinical guidance from the anticoagulation forum. J Thromb Thrombolysis 2020; 50(1):72-81. doi:10.1007/s11239-020-02138-z

39. Moores LK, Tritschler T, Brosnahan S, et al. Prevention, diagnosis and treatment of venous thromboembolism in patients with 
COVID-19: CHEST guideline and expert panel report. Chest 2020; Jun. doi:10.1016/j.chest.2020.05.559

40. Dabbagh MF, Aurora L, D'Souza P, Weinmann AJ, Bhargava P, Basir MB. Cardiac tamponade secondary to COVID-19. JACC Case Reports 2020; Apr 23. doi:10.1016/j.jaccas.2020.04.009

41. Hébert PC, Wells G, Blajchman MA, et al. A multicenter, randomized, controlled clinical trial of transfusion requirements in critical care. N Engl J Med 1999; 340(6):409-417. doi:10.1056/ NEJM199902113400601

42. Fox S, Dugar S. Point-of-care ultrasound and COVID-19. Cleve Clin J Med 2020; May 14. doi:10.3949/ccjm.87a.ccc019

Correspondence: Siddharth Dugar, MD, Department of Pulmonary Medicine, Respiratory Institute, L22-0330, Cleveland Clinic, 9500 Euclid Avenue, Cleveland, OH 44195; dugars@ccf.org 